\title{
IRAS 0421+0400: A CURIOUS SPIRAL WITH FLARING JETS
}

\author{
W. STEFFEN AND A.J. HOLLOWAY \\ Department of Physics and Astronomy, University of Manchester, \\ Manchester M13 9PL, UK
}

AND

A. PEDLAR AND D.J. AXON

Nuffield Radio Astronomy Laboratories, University of Manchester, Jodrell Bank, Macclesfield, Cheshire SK11 9DL, UK

\begin{abstract}
We present new VLA observations of IRAS $0421+0400$ at 1.4 and $5 \mathrm{GHz}$, providing dual frequency information and higher resolution than previously available. We find extremely accurate alignment of the central double with the closest (though not brightest) feature in the southern kiloparsec hotspot region. There is a tight relation between the symmetric radio structure and emission line gas in the hotspot regions of this Seyfert 2 type galaxy.
\end{abstract}

The emission lines in the active galaxy IRAS $0421+0400$ show a dramatic $\left(\approx 900 \mathrm{kms}^{-1}\right)$ increase in the velocity spread at the position of radio hotspots which are located at the beginning of extended radio lobes (Hill et al, 1988; Holloway et al, 1996). In Holloway et al (1996) and Steffen et al (1996) we have investigated a model of a jet crossing a shocked boundary between the ISM/IGM-interface (Loken et al, 1995, and references therein) which could explain the peculiar spectrum and the flaring of the radio structure at approx. 5 arcsec on either side of the galaxy. In this paper we present preliminary results from new VLA observations at 1.4 and $5 \mathrm{GHz}$, providing dual frequency information and higher resolution than previous observations (Beichman et al, 1985; Hill et al, 1988). The observations were performed on September $1^{s t}, 1995$, with the VLA in A-array during a 4 hour run.

We find that the hypothetical jets connecting the centre of the galaxy with the lobes stays invisible at the low frequency of $1.4 \mathrm{GHz}$ down to a level of $0.15 \mathrm{mJy} /$ beam. The structure of the southern hotspot region is 

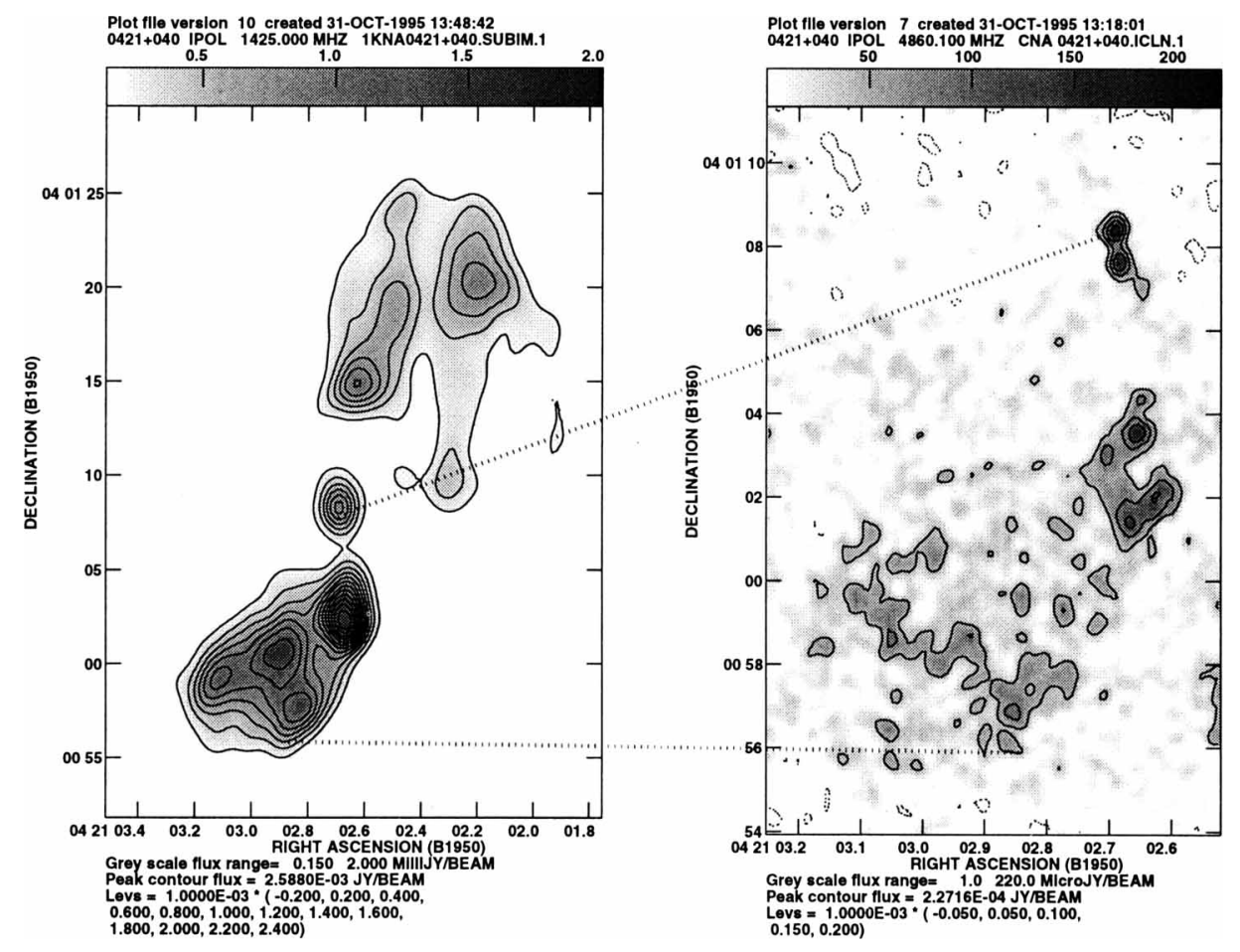

Figure 1. VLA A-array images of IRAS $0421+0400$ at $1.4 \mathrm{GHz}$ (left) and $5 \mathrm{GHz}$. The beamsizes are $1.78 \times 1.48$ and $0.52 \times 0.43$ arcsec $\left(P A=-9.5^{\circ}\right)$, respectively. 1 arcsec corresponds to $0.9 \mathrm{kpc}(\mathrm{h}=1)$.

well resolved at $5 \mathrm{GHz}$ and is found to be very complex consisting of a bright unresolved hotspot and several filaments. The central double source is extremely well aligned (to within $\left\langle 1^{\circ}\right.$ ) with an unresolved low brightness feature located at approximately 1 arcsec inwards from the southern main hotspot. The northern radio emission is consistently of less flux density and no correspondingly well aligned features is found in the northern region.

\section{References}

Beichman C., Wynn-Williams C.G., Lonsdale C.J., et al. (1985), ApJ, 293, p.148

Hill G.J., Wynn-Williams C.G., Becklin E.E., MacKenty J.W. (1988), ApJ, 335, p.93

Holloway A.J., Steffen W., Pedlar A., et al. (1996), MNRAS, in press

Steffen W., Holloway A.J., Pedlar A., Axon D.J., (1996), Workshop on Energy Transport in Radio Galaxies \& Quasars, Astronomical Society of the Pacific Conference Series, in press

Loken C., Roettiger K., Burns J.O., Norman M. (1995), ApJ, 445, 80 